\title{
SCREENING OF PATHOPHYSIOLOGICAL MECHANISMS AND PSYCHOLOGICAL AND OCCUPATIONAL FACTORS, DETERMINING RISK OF CARDIOVASCULAR DISEASES IN HIGH-RISK PROFESSIONS
}

\author{
R. Nikolova', S. Danev², T. Kundurjiev', N. Madjarov' \\ ${ }^{1}$ Department of Occupational Medicine, Faculty of Public Health, Medical University - Sofia, Bulgaria \\ ${ }^{2}$ Medeia Group Ltd. Company - Sofia, Bulgaria
}

\begin{abstract}
Cardiovascular diseases (CVD) are among the most significant and common chronic non-communicable diseases in Bulgaria. Screening, identification and registration of pathophysiological mechanisms and psychological and occupational factors related to CVD should be implemented in the diagnostic work-up. Job analysis contributes to the implementation of screening and identification of the most significant psychological and professional factors related to the work activity. Aim: The aim of our study is to examine the pathophysiological mechanisms of autonomic cardiovascular control and psychological and occupational factors determining the risk of CVD. Materials and methods: The following groups of individuals were examined: 83 physicians, 54 nurses, 23 employees and 40 militaries. The autonomic cardiovascular control was studied through the timeand frequency-domain based measures of the Analysis of Heart Rate Variability (HRV). Results: The results of the ANOVA test showed that there was a statistically significant difference in the time-domain and frequency-domain based HRV measures between the compared groups: $X[F(4 ; 225)=4.455, p=0.001]$; $\operatorname{SDNN}[F(4 ; 225)=3.876, p=0.005]$; SDNN5 [F $(4 ; 225)=3.083, p=0.01] ; p N N 50[F(4 ; 225)=8.303, p<0.001] ;$ rMSSD [F (4; $225)=8.711, p<0.001] ; \operatorname{SDSD}[F(4 ; 225)=8.323, p<0.001 ; \operatorname{VLF}[F(4 ; 225)=1.204, p$ $=0.31] ; \operatorname{LF}[F(4 ; 225)=3.874, p=0.005] ; \operatorname{HF}[F(4 ; 225)=7.790, p<0.001]$ and $L F / H F[F$ $(4 ; 225)=8.601, p<0.001]$. The results of our study revealed that occupational stress and cognitive workload in physicians, nurses, and employees induced a process of reciprocally combined inhibition of the parasympathetic branch of the autonomic nervous system (ANS) (SDNN, SDNN5, pNN50, rMSSD, and HF) and activation of the sympathetic branch of ANS (LF), while in military personnel they cause a process of co-activation of the parasympathetic and sympathetic branches of the ANS (rMSSD, SDNN, SDNN5, pNN50 and LF/HF). Conclusion: Stress-induced dysfunctional autonomic cardiovascular regulation, pathophysiological control mechanisms, psychological and occupational factors determine the risk of CVD. Their identification could contribute to the diagnostics and prevention of CVD.
\end{abstract}

Key words: cardiovascular diseases; screening; occupational risk factors; psychological risk factors; Heart Rate Variability; autonomic cardiovascular control; job analysis

Corresponding author: Assoc. Prof. Rouja Nikolova, MD, PhD, Department of Occupational Medicine, Faculty of Public Health, Medical University - Sofia, 8 "Byalo More" Street, 1527 Sofia, Bulgaria, e-mail: nikolovarouja@yahoo.com 


\section{INTRODUCTION}

dentifying the risk factors of cardiovascular diseases (CVD) is extremely important for our time and for our research work. It could contribute to the identification and prevention of CVD, as they represent a significant percentage of chronic non-communicable diseases in Bulgaria. We have focused our attention on the screening of physiological and pathophysiological mechanisms determining the pattern and mode of autonomic cardiovascular control in the conditions of chronic stress exposure. Stress-induced dysfunctional cardiovascular regulation and the resulting pathophysiological mechanisms determine the risk of developing CVD. Of parallel research interest is the screening of significant occupational, psychological and social factors inducing and exacerbating the stress response. We have focused on the modern high-risk professions of workers in significant and risky areas of socio-political life such as: medical professionals - physicians and nurses, military personnel and civil servants - professions, characterized by markedly high magnitude of stress and cognitive workload.

Chronic exposure to constantly persistent occupational health risk factors such as stress and cognitive workload may induce a process of change in autonomic cardiovascular control, which we study and assess through the indicators of the Analysis of Heart Rate Variability (HRV) [1-7]. The advantages of HRV as a main methodological method and instrument developed, researched and applied in the fields of physiology, psycho-physiology, cardiology, preventive and occupational medicine are that it may screen and diagnose the major physiological and pathophysiological mechanisms controlling the cardiovascular regulation: sympathetic-parasympathetic autonomic cardiovascular control and baroreceptor modulation of heart rhythm [8-11]. Under the influence of stress dictated by an increase in the degree of cognitive workload, the autonomic cardiovascular control is disrupted by developing a dysfunctional model of regulation in which the control mechanisms change with a tendency to alteration of the activity of both branches: sympathetic and parasympathetic of the Autonomic Nervous System (ANS). The creation and development of the Doctrine for Autonomic Space [12] shows that the two branches of the Autonomic Nervous System (ANS) are not only reciprocally connected and combined. Rather, their activity may not be interrelated (co-activation or co-inhibition) or even incompatible (a change in the activity of one branch may occur or intervene independently of the activity of the other branch).

In order to assess the stress and the cognitive workload of the professions, which are distinguished and characterized by high risk in the process of work performance, we conduct and carry out parallel screening of occupational and psychological factors through another work-physiological method such as Job Analy- sis [13]. Job analysis contributes to register the most significant occupational and psychological risk factors that induce and exacerbate the stress response as a result to increased magnitude of cognitive load. In the last decades of the XXI century, significant changes have taken place in the professional and psychological characteristics of work activity and their social connection, closely related to the organization and management of work, resulting in risks and new challenges in the field of occupational health and safety. The psychological, social and occupational risks of specific jobs are identified as significant risks. The topic of occupational work-related stress is closely linked to occupational and psychosocial risks and is widely accepted as a significant challenge in the field of public health and safety. The work environment and job characteristics are important factors influencing a person's health status. Significant changes occurring in the field of employment cause and result in the strengthening of existing and the emergence of new risks and challenges in the field of public health. Psychological, social and occupational risks in the workplace are identified as new significant risks, stressors and hazards. Complicated occupational and psychological risks and situations in the modern workplace in high-risk occupations impair the functional state of the cardiovascular system (CVS), assessed by HRV and potentiate the pathophysiological mechanisms of autonomic cardiovascular control that may induce CVD risk.

The aim of our study is to examine the pathophysiological mechanisms of autonomic cardiovascular control and psychological and occupational factors determining the risk of CVD. Their identification could contribute to the diagnostics and prevention of CVD.

\section{MATERIALS AND METHODS}

Four groups of individuals were included and examined in our study: physicians, nurses, employees, and military personnel. Physicians and nurses who participated in the study worked at the University First General Hospital for Active Treatment - Sofia „St. John the Baptist” Ltd. and at UMHAT „Tsaritsa Joanna - ISUL“, in the Departments of Intensive Cardiology, Clinical Therapeutics, Surgery, Neurology and in the Emergency Department. The employees worked in the Administration of the Diplomatic Property Agency in the Country (ADIS). The military personnel worked in the field of the Bulgarian Military Forces.

The group of physicians consisted of 83 individuals (mean age/SD, 44.06/12.77 years; average job tenure/SD, 18.96/0.30 years), of which 40 men and 43 women. The group of nurses consisted of 54 women (mean age/SD, 44.89/10.97 years; average job tenure/ $\mathrm{SD}, 23.85 / 1.00$ years). The group of employees consisted of 23 individuals (average age/SD, 45.91/8.44 years; average job tenure/SD, 22.91/8.44 years), of which 15 men and 8 women. The group of military per- 
sonnel consisted of 40 individuals (average age/SD, 32.08/10.37 years; average job tenure/SD, 10.80/9.02 years), of which 38 men and 2 women.

The study complied with the ethical standards set out in the Declaration of Helsinki of experimental studies in humans and has been approved by the Ethics Committee for Scientific Research at the Medical University of Sofia.

\section{Job Analysis}

A detailed job analysis has been applied and performed to investigate and determine the risk factors inherent in the professional activity in the groups studied in high-risk professions [13]. The purpose of the extensive analysis of the work activity was to screen and identify the major risk factors by: 1) conducting an interview with the employer and the employees about the nature and character of the work; 2) monitoring the work and performance of work tasks; 3) analyzing the emerging psycho-social components and characteristics of work activity that exist in the working place.

Investigation of the functional state of the cardiovascular system (CVS) and autonomic cardiovascular control, using a computer diagnostic method and a system for Analysis of Heart Rate Variability.

A computer diagnostic method and a system for Analysis of Heart Rate Variability (HRV) have been applied to study the functional state of CVS and autonomic cardiovascular control [14-16]. HRV measures were determined by 10-minute ECG recordings between 9-11 a.m. in a sitting position after a one-hour rest period.

The following time-domain based and frequency-domain based measures of HRV were analyzed:

\section{Time-domain based HRV measures:}

X (mean RR intervals) (msec), respectively mean heart rate (beats/min); SDNN (standard deviation of normal NN RR intervals) (msec); SDNN5 (standard deviation at 5 consecutive RR intervals) (msec); pNN50 (percentage of difference between adjacent NN RR intervals that is greater than $50 \mathrm{msec}$ ) (\%); rMSSD (root mean square difference between two consecutive normal RR intervals) (msec); SDSD (standard deviation of the differences between adjacent NN RR intervals) (msec).

\section{Frequency-domain based HRV measures:}

VLF (spectral power of the RR intervals in the verylow frequency band) (0.0033-0.04 H Hz) (ms2) (reflects thermoregulation and renni-angiotensinaldosterone activity); LF (spectral power of RR intervals in the low-frequency band) (0.04-0.15 Hz) (ms2) (some authors believe that it is sympathetic mediated, and others that it reflects sympathetic and parasympathetic activity, and baroreceptor modulation and activity); HF (spectral power of the $\mathrm{RR}$ intervals in the high-frequency band) (0.15$0.4 \mathrm{~Hz}$ ) ( $\left.\mathrm{ms}^{2}\right)$ (parasympathetically mediated); LF/
HF (ratio of the spectral power of the RR intervals in the low-frequency band to the high-frequency band) (\%) (ratio of the sympathetic to parasympathetic activity).

\section{Data analysis}

Means of HRV measures between: studied groups were compared by independent t-test and analysis of variance.

\section{RESULTS}

Occupational-physiological screening of basic and major occupational and psychological risk factors as a result of the investigation of working activity was performed through Job Analysis. Following psychological and occupational factors were observed in high-risk professions:

\section{EMPLOYESS:}

\subsection{PSYCHOLOGICAL FACTORS}

- psychosocial stress caused by exposure to psychosocial risks

- psychological load related to the performance of emergency, urgent and instant tasks;

1.2. OCCUPATIONAL FACTORS:

- neuro-psychological load

- requirements for high responsibility when working with foreign citizens and clients, and the good and correct presentation of our country to them;

- high concentration and distribution of attention in the performance of work;

- visual load.

2. MILITARY PERSONNEL

\subsection{PSYCHOLOGICAL FACTORS}

- psychological load associated with the risk of terrorism;

- psychological and social load related to: deployment in new environment; provocations; work on duties; consideration of way of life, customs, and religion of the native population; separation from family; limited access to civilian places;

- psychological load related to risky emergencies and critical situations;

- psychological load associated with the risk of neglect of the human factor and the risk of accidents;

- psycho-social stress caused by exposure to psycho-social risks.

\subsection{OCCUPATIONAL FACTORS}

- neuro-psychological load

- mental workload

- requirements for high responsibility for the life of the population;

- requirements for conducting life-saving operations, missions and operations;

- security and safety requirements;

- potentially traumatic combat situations: combat attacks; dangerous patrols; safeguarding important objectives; increased risk of escalating conflicts; 
- work in conditions of time stress;

- requirements for fast and urgent expeditious intervention;

- high concentration and distribution of attention in the performance of work;

- visual, sound and hearing load.

\section{PHYSICIANS:}

\subsection{PSYCHOLOGICAL FACTORS:}

- psychosocial stress caused by exposure to psychosocial risks;

- psychological load related to risky emergencies, and critical situations in conditions of resuscitation and intensive intervention in saving lives and providing therapeutic intervention;

- psychological load associated with the risk of neglect of the human factor and the risk of accidents;

3.2. OCCUPATIONAL FACTORS:

- neuro-psychological workload;

- cognitive workload;

- requirements for high responsibility for the health and life of patients, and responsibility to the society and to patients' loved ones;

- requirements for life-saving operations and surgeries requiring high risk and critical intervention;

- diagnosis, treatment and care of patients in an emergency and in diagnostically and therapeutically difficult cases;

- work in conditions of time stress;

- security and safety requirements;

- requirements for multiple qualifications and skills;

- requirements for rapid and urgent expeditious intervention;

- shift work;

- high concentration and distribution of attention when performing the work activity;

- involvement of higher thought and associative processes;

- mobility of memory involvement processes;

- visual, sound and auditory load.

\section{NURSES:}

\subsection{PSYCHOLOGICAL FACTORS:}

- psychosocial stress caused by exposure to psychosocial risks

- psychological stress associated with the risk of neglect of the human factor and the risk of accidents.

4.2. OCCUPATIONAL FACTORS:

- neuro-psychological load;

- requirements for high responsibility for the health and life of patients;

- emergency patient care and diagnostically and therapeutically difficult cases;

- security and safety requirements;

- requirements for fast and urgent expeditious intervention;

- shift work;
- high concentration and distribution of attention in the performance of work;

- visual, sound and hearing load.

Diagnostics of the functional state of CVS and screening of the most essential physiological and pathophysiological mechanisms determining the risk of CVD:

The ANOVA test revealed that there was a statistically significant difference in the time-domain based HRV measures between the compared groups: $X$ [F (4; $225)=4.455, p=0.001] ; \operatorname{SDNN}[F(4 ; 225)=3.876, p$ $=0.005]$; SDNN5 [F $(4 ; 225)=3.083, p=0.01]$; pNN50 $[F(4 ; 225)=8.303, p<0.001] ; \operatorname{rMSSD}[F(4 ; 225)=$ 8.711, $p<0.001]$; SDSD [F $(4 ; 225)=8.323, p<0.001]$.

We observed that the mean value of rMSSD was significantly increased in military personnel compared to employees, physicians, and nurses. The high stress resistance and physical training characterizing the group of military personnel had a favorable effect on the high mean values of parasympathetically-mediated time-domain based HRV measures: SDNN, SDNN5, pNN50 and rMSSD distinguishing them from those of the groups of employees, physicians, and nurses which had reduced parasympathetically-mediated values.

The results of the ANOVA test showed that there was a statistically significant difference in the frequencydomain based HRV measures between the compared groups: VLF $[F(4 ; 225)=1.204, p=0.31] ;$ LF $[F(4 ; 225)=3.874, p=0.005] ; \mathrm{HF}[F(4 ; 225)=7.790$, $\mathrm{p}<0.001]$ and LF/HF $[F(4 ; 225)=8.601, p<0.001]$.

Autonomic cardiovascular control studied by HF, which reflects parasympathetic activity, was significantly reduced in the groups of employees, military personnel, physicians and nurses, which is an indicator of the eliciting and developing of a stress response in these groups. Decreased activity in the area of vagally-mediated HF correlates with stress. The results also showed that baroreceptor modulation of the heart rate studied by parasympathetically and sympathetically-mediated LF was significantly reduced in the groups of employees, physicians and nurses compared to military personnel. Another result indicates that the LF/HF ratio - an indicator of the ratio of autonomic sympathetic-parasympathetic tone to parasympathetic activity was significantly increased in military personnel compared to groups of physicians and nurses, and that it was also significantly increased in employees.

\section{DISCUSSION}

Occupational-physiological screening of occupational, psychological and social risk factors and factors induced by the work environment of individuals working in nationally responsible systems and areas of sociopolitical life (health, defense and public administration) contributes to early identification and detection of determinants of occupational stress. The results of our study 
indicate that the occupational, psychological and social risk factors to which the investigated individuals working in these high-risk occupations are exposed, contribute to modern determinants of occupational stress. The risk factors of physicians and military personnel prevail compared to nurses and employees. In the process of our work we strive to apply new modern computerized methods for medical diagnostics and relevant psychological methods that promote early identification of risk of CVD. Significant changes occurring in the field of employment result in the emergence of new risks and challenges in the field of public health, especially in the group of physicians and military personnel. Psychological, social and occupational risks in the workplace are identified as new significant risk factors and stressors. The modern determinants of occupational stress in the studied high-risk professions found by us impair the functional state of CVS, assessed by HRV and potentiate the pathophysiological mechanisms of autonomic cardiovascular control that induce risk of CVD.

In the military personnel our results demonstrated a process of co-activation of the sympathetic and parasympathetic branches of the ANS. The high stress resistance and physical training characterizing this group have a favorable effect on the high mean values of parasympathetically-mediated time-domain based HRV measures: SDNN, SDNN5, pNN50 and rMSSD, and as a result of stress exposure a process of activation of sympatethic branch is observed, evaluated by the frequency-domain based HRV measure - LF/HF, which is an indicator of the ratio of baroreceptor modulation of heart rate with dominance of sympathetic activity to parasympathetic.

In support of our result and statement is the finding that stressors from the work environment cause a process in the Central Nervous System (CNS), in the frontal lobes of the brain, which determines whether the activity takes place in the frontocortical-medulla oblongata cerebral pathway. The process in this neurocerebral pathway leads to double autonomic activity of both branches of the ANS and inhibition of the mechanisms of homeostasis. Efferent autonomic activity alone or in combination with myocardial infarction triggers the initiation of ventricular fibrillation [17-18].

In the group of physicians, nurses and employees we observed a process of inhibition of the parasympathetic branch of the ANS, assessed by parasympathetically-mediated: time-domain based measures SDNN, SDNN5, pNN50, rMSSD and the frequency-domain based HF measure as this process is a characteristic and typical result due to stress exposure. The baroreceptor modulation of the heart rhythm studied by parasympathetically and sympathetically-mediated LF was reduced in the groups of physicians, nurses and employees with a tendency to dominate sympathetic activity. These results coincide with the established relationship between occupational stress and the components of HRV, which suggests that occupa- tional stress causes vagal withdrawal and sympathetic saturation, indicating that the prevalence of sympathetic mechanisms leads to cardiac electrical instability [19]. Similar results of sympathetic hyperreactivity and parasympathetic inhibition due to stress exposure were found by Kageyama et al. in 1998 [20].

In summary, occupational stress in physicians, nurses and employees induces a process of reciprocally combined inhibition of the parasympathetic branch of the ANS and activation of the sympathetic branch of the ANS, while in military personnel it causes a process of co-activation of the parasympathetic and sympathetic branches of the ANS. Dysfunctional autonomic cardiovascular control develops in which the physiological mechanisms of control are disturbed and pathophysiological mechanisms are formed. This process occurs due to the exposure of risk psychological and occupational factors, which, as already emphasized, are the determinants of occupational stress in high-risk occupations. The tendency and mode of dysfunctional autonomic cardiovascular control as a result to the impact of occupational stress contributes to the determination of CVD risk [21-26].

Job analysis contributes to the implementation of screening and identification of the most significant psychological and professional factors related to the work activity. These risk factors appear to be determinants of occupational stress. Moreover, as a result of the induced stress response, a pattern of dysfunctional autonomic cardiovascular control develops. The exposure to existing psychological and occupational risk factors and the emergence of new dangers, risk factors and challenges result in the disturbance of the physiological mechanisms of control and formation of pathophysiological mechanisms.

\section{CONCLUSIONS}

Job analysis facilitates occupational-physiological screening of occupational, psychological and social risk factors at high-risk professions. The percentage of risk factors is the highest among physicians and military personnel, but there is also an exposure to such factors among nurses and employees.

Job analysis and Heart Rate Variability are reliable physiological methods for discrimination of occupational, psychological risk factors and physiological, and pathophysiological mechanisms of autonomic cardiovascular control [27-29]. Job analysis of work activity could define some of the most basic determinants of occupational stress.

Chronic exposure to occupational stress caused dysfunctional autonomic cardiovascular control. In physicians, nurses and employees a process of reciprocally combined inhibition of the parasympathetic branch of the Autonomic Nervous System (ANS) and activation of the sympathetic branch of the ANS was induced. However, among the military personnel a 
process of co-activation of the parasympathetic and sympathetic branches of the ANS developed.

Stress-induced dysfunctional autonomic cardiovascular control and the established pathophysiological control mechanisms, as well as the determination of some of the most significant and substantial determinants of occupational stress contribute to the definition of CVD risk.

The mode and pattern of stress-induced dysfunctional autonomic cardiovascular control are different among individuals from different professions. This finding could be used in the future as part of the prevention strategies and early identification of the CVD risk among these high-risk professions.

\section{Acknowledgements}

This research is related to the project „Analysis and assessment of occupational psycho-social stress and cardiovascular risk in individuals working in significant and risk spheres of socio-political life", funded by the Council of Medical Sciences of the Medical University of Sofia, GRANT 2018, Contract № D-94/03.05.2018 and Project № 7855/23.11.2017.

Disclosure summary: The authors have nothing to disclose.

\section{REFERENCES}

1. Sommerfeldt S, Schaefer S, Brauer M. et al. Individual Differences in the Association Between Subjective Stress and Heart Rate Are Related to Psychological and Physical WellBeing. Psychol Sci. 2019, 30(7):1016-1029.

2. Järvelin-Pasanen $S$, Sinikallio $S$, Tarvainen $M$. Heart rate variability and occupational stress - systematic review. Ind Health. 2018, 56(6):500-511.

3. Clough B, March S, Chan R. et al. Psychosocial interventions for managing occupational stress and burnout among medical doctors: a systematic review. Syst Rev. 2017, 6:144.

4. Borchini R, Veronesi G, Bonzini M. et al. Heart Rate Variability Frequency Domain Alterations among Healthy Nurses Exposed to Prolonged Work Stress. Int J Environ Res Public Health. 2018, 15(1):113.

5. Theorell T. A long-term perspective on cardiovascular job stress research. J Occup Health. 2019, 61(1):3-9.

6. Rodrigues S, Paiva J, Dias D. et al. Cognitive Impact and Psychophysiological Effects of Stress Using a Biomonitoring Platform. Int J Environ Res Public Health. 2018, 15(6):1080.

7. Sara J, Prasad M, Eleid M. et al. Association Between WorkRelated Stress and Coronary Heart Disease: A Review of Prospective Studies Through the Job Strain, Effort-Reward Balance, and Organizational Justice Models. J Am Heart Assoc. 2018, 7(9):e008073.

8. Iliescu R, Tudorancea I, Irwin E. et al. Chronic baroreflex activation restores spontaneous baroreflex control and variability of heart rate in obesity-induced hypertension. Am J Physiol Heart Circ Physiol. 2013, 305(7):H1080-H1088.

9. Ben-Tal A, Shamailov S, Paton J. Central regulation of heart rate and the appearance of Respiratory Sinus Arrhythmia: new insights from mathematical modeling. Math Biosci. 2014, 255:71-82.

10. Park J, Marvar P, Liao P. et al. Baroreflex dysfunction and augmented sympathetic nerve responses during mental stress in veterans with post-traumatic stress disorder. J Physiol. 2017, 595(14):4893-4908.

11. McCraty R, Shaffer F. Heart Rate Variability: New Perspectives on Physiological Mechanisms, Assessment of Self-reg- ulatory Capacity, and Health risk. Glob Adv Health Med. 2015, 4(1):46-61.

12. Berntson G, Cacioppo J. and Quigley K. Autonomic determinism: The modes of autonomic control, the doctrine of autonomic space, and the laws of autonomic constraint. Psychol. Review, 1991, 4, 459-487.

13. Landau K, Rohmert W (Eds.), Recent developments in job analysis. London, Taylor \& Francis, 1989.

14. Danev S. Informativeness of the heart rhythm in occupational-physiological aspect. Dissertation: Doctor of Medical Sciences. National Center for Hygiene, Medical Ecology and Nutrition. Sofia, 1989 (In Bulg.).

15. Nikolova R. Approbation of the method for Analysis of Heart Rate Variability in models for neuro-sensorial occupational stress and its methodological improvement. Dissertation: National Center for Hygiene, Med Ecol Nut Sofia, 1993 (In Bulg.).

16. Danev S. VitalScan ANS - Software-hardware method for Analysis of Heart Rate Variability. Medeia Ltd., Vancouver, 2016.

17. Skinner J. Regulation of cardiac vulnerability by the cerebral defense system. J. Am. Coll. Cardiol., 1985, 5, 88B-94B.

18. Wolf $\mathrm{S}$. The environment-brain-heart connection: econeurocardiology. Occup. Med., 2000, 15, 107-109.

19. Malik M, Camm A. Components of heart rate variability $p$ what they really mean and what we really measure. Am. J. Cardiol., 1993, 72, 821-822.

20. Kageyama T, Nishikido N, Kobayashi T. et al. Long commuting time, extensive over time, and sympathodominant state assessed in terms of short-term heart rate variability among male collar workers in the Tokyo megapolis. Ind. Health, 1998, 36, 209-217.

21. Kubota Y, Chen L, Whitsel E. et al. Heart Rate Variability and Lifetime Risk of Cardiovascular Disease: the Atherosclerosis Risk in Communities Study. Ann Epidemiol. 2017, 27(10):619-625.

22. Benjamin E, Blaha M, Chiuve S. et al. Heart Disease and Stroke Statistics - 2017 Update: A Report From the American Heart Association. Circulation. 2017, 135(10):e146-e603.

23. Go A, Mozaffarian D, Roger V. et al. Heart Disease and Stroke Statistics - 2014 Update: A Report From the American Heart Association. Circulation. 2014, 129(3):e28-e292.

24. Stein P, Barzilay J, Chaves P. et al. Novel Measures of Heart Rate Variability Predict Cardiovascular Mortality in Older Adults Independent of Traditional Cardiovascular Risk Factors: The Cardiovascular Health Study (CHS). J Cardiovasc Electrophysiol. 2008 Nov; 19(11): 1169-1174.

25. Piepoli M, Hoes A, Agewall S. et al. 2016 European Guidelines on cardiovascular disease prevention in clinical practice: The Sixth Joint Task Force of the European Society of Cardiology and Other Societies on Cardiovascular Disease Prevention in Clinical Practice (constituted by representatives of 10 societies and by invited experts) Developed with the special contribution of the European Association for Cardiovascular Prevention \& Rehabilitation (EACPR). Eur Heart J. 2016, 37(29):2315-2381.

26. Khan H, Kunutsor S, Kalogeropoulos A. et al. Resting Heart Rate and Risk of Incident Heart Failure: Three Prospective Cohort Studies and a Systematic Meta-Analysis. J Am Heart Assoc. 2015, 4(1):e001364.

27. de Looff P, Cornet L, Embregts P. et al. Associations of sympathetic and parasympathetic activity in job stress and burnout: A systematic review. PLoS One. 2018, 13(10):e0205741.

28. Borchini R, Veronesi G, Bonzini M. et al. Heart Rate Variability Frequency Domain Alterations among Healthy Nurses Exposed to Prolonged Work Stress. Int J Environ Res Public Health. 2018, 15(1):113.

29. Cao X, MacNaughton $P$, Cadet L. et al. Heart Rate Variability and Performance of Commercial Airline Pilots during Flight Simulations. Int J Environ Res Public Health. 2019, 16(2):237.

Received: 15 July 2020, Revised: 20 September 2020 Accepted: 23 September 2020 